\title{
Community-based clinical teaching
}

\author{
Christopher D. Lynch, Peter J. Ash, Barbara L. Chadwick \\ and Elizabeth T. Treasure
}

Cardiff

The practice of dentistry is continuously evolving and is developing at a considerable pace. Even setting aside the need to keep up to date with the ever-increasing range of clinical techniques and skills, the management of patients and the many administrative issues facing dental practices, contemporary dental practitioners encounter many challenges in their dayto-day work. How can new and young dentists be best prepared for professional careers in this environment?

For dental educators, the common challenge is to prepare graduating students for eventual careers as independent dental practitioners. This is a significant challenge, typically when dental school programmes are often criticised for 'lagging behind' developments in clinical practice. Our dental schools of 2009 face many barriers that affect the delivery of dental student teaching programmes, of which one is the difficulty of accessing suitable 'primary care' patients. Traditionally, dental students undertook patient management within the environment of a dental school, where under supervision they completed clinical tasks and exercises, with a focus on requirements and quotas and in more modern times on competencies. While operating in this educationally valuable, but ultimately artificial environment, new dental school graduates were often unprepared for the rigors and demands of running an efficient and well-organised dental practice while at the same time delivering high quality dental treatment for patients and their families within a primary care environment.

\section{FOCUSSING ON ORAL HEALTHCARE PROGRAMMES}

In response to these challenges, community-based clinical teaching programmes (sometimes termed 'outreach dental teaching') began to develop internationally and in certain UK dental schools, most notably over the past ten years. The aim of these community-based clinical teaching programmes is to facilitate the transition of dental undergraduate students and student dental care professionals from a dental school environment - often located within a secondary care setting - to a unit where they are more closely integrated with what they will encounter in vocational training and subsequently in independent practice in a primary care setting. In so doing, student learning is more focussed on the delivery of an oral healthcare programme for an individual patient within a primary care setting, rather than being simply centred on the completion of prescribed clinical tasks with the aim of achieving quotas or requirements. The development of student learning within community-based clinical teaching centres complements the aims of government policies, such as Options for change which recommends that dental education should centre on developing skills needed in practice, with greater involvement of primary care teaching centres.

UK dental schools have approached the development of community-based clinical teaching in a variety of ways. Some schools, such as the celebrated Sheffield programme, disperse their students across a number of selected dental practices. Other dental schools, such as our own at Cardiff, and more recently in Leeds, have developed purpose-built units, where patients can be seen in a primary care setting. At Cardiff, a twelve-chair unit was opened at the St David Hospital in 2002, while a similar eighteen-chair unit is currently being developed at Mountain Ash; both being sited in areas with high need for primary care dentistry. In contrast to other schools where outreach schemes are limited to discrete areas of clinical teaching such as paediatric or restorative dentistry, the Cardiff model allows dental students to provide 'total patient care' across the range of primary care dentistry. Student feedback, and the reported improvement in student confidence in managing patients and delivering dental treatments are exceedingly positive. We are happy and confident that our approach will help our students as they develop in their careers to become highly-skilled and competent independent clinicians.

But what of the future? In educational circles, it is axiomatic that 'assessment drives learning'; or in other words that students of any discipline choose to learn material that will be expected to feature in forthcoming assessments and examinations. This principle is often observed in dental education, but it is also true that dental students, being clever and intelligent individuals and committed to their patients, are also focussed on the 'assessments' they will encounter every day in their working careers as individual and independent providers of quality oral healthcare programmes. Outreach/community-based clinical teaching offers a realistic and valuable approach to preparing for such environments. On 29 April 2009, leading teachers of outreach dentistry met at Cardiff to affirm their commitment to, and to recognise the role of and importance of outreach and community-based clinical teaching programmes in contemporary dental school educational programmes. Community-based clinical teaching has been a significant dental educational development over the past ten years - who knows what the next ten will bring?

DOI: 10.1038/sj.bdj.2009.730 